\title{
Brain FDG-PET: clinical use in dementing neurodegenerative conditions
}

\author{
Flavio Nobili ${ }^{1}$ (D) $\cdot$ Reinhold Schmidt $^{2} \cdot$ Ignasi Carriò $^{3} \cdot$ Giovanni B. Frisoni $^{4}$
}

Published online: 23 April 2018

(C) Springer-Verlag GmbH Germany, part of Springer Nature 2018

For several years now clinicians have used brain FDG-PET to guide the clinical diagnosis of dementing disorders. Especially neurologists, but psychiatrists and geriatricians as well, ask for FDG-PET when they are uncertain about their diagnostic hypothesis, more often after neuropsychological assessment and brain magnetic resonance imaging.

However, neither the submitting physician nor the nuclear medicine physician (NMP) have comprehensive clinical guidelines or recommendations available on when and why to use FDG-PET in neurodegenerative diseases, and often the choice relies on how much one or the other 'trusts' in the exam. Some indications are included in the European Association of Nuclear Medicine (EANM) procedure guidelines [1] and some more structured ones are contained in the European Federation of Neurological Societies (EFNS) guidelines to the use of neuroimaging in dementia [2], but neither of the two covers all the key aspects of the topic, for instance, the cognitive impairment in Parkinson's disease, Huntington's disease (HD), amyotrophic lateral sclerosis (ALS), progressive supranuclear palsy (PSP), and the issue of semi-quantification.

With these limitations in mind, the Neuroimaging Committee (NIC) of the EANM thought at the beginning of 2014 that the time had come to prepare more structured and comprehensive recommendations, to be shared with the main

Flavio Nobili

flaviomariano.nobili@hsanmartino.it

1 Department of Neuroscience (DINOGMI), University of Genoa and Neurology Clinics, IRCCS Polyclinic San Martino Hospital, Genoa, Italy

2 Department of Neurology, Medical University of Graz, Graz, Austria

3 Department of Nuclear Medicine, Autonomous University of Barcelona, Hospital Sant Pau, Barcelona, Spain

4 LANVIE (Laboratoire de Neuroimagerie du Vieillissement), Department of Psychiatry, University of Geneva,

Geneva, Switzerland clinical Society in the field, i.e., the European Academy of Neurology (EAN), formerly EFNS. The EAN Offices immediately gave a positive answer and the work started.

The first main step was identifying a group of experts in the field, on behalf of the two Societies, who were chosen among the components of the EANM NIC (four people) and the EAN neuroimaging and dementia study groups (three people). Without entering the details, we have to say that we had to overcome a series of issues, ranging from the need of finding a group of 'facilitators' in organizing literature evidence and guiding Delphi Consensus to the need of harmonizing different cultures between NMPs and neurologists. The job took 3 years, from 2015 to the end of 2017. Preliminary data were presented during the II EAN Congress in Amsterdam, June 2017 [3-6].

The main recommendation paper was prepared for submission to the European Journal of Neurology, but we soon realized that it could not include all the data collected during the analysis and assessing of literature. Thus, we proposed the European Journal of Nuclear Medicine and Molecular Imaging (EJNMMI) a special issue containing the detailed results of the data search. The Editor in Chief Prof. Ignasi Carriò favorably accepted the proposal during the 2016 EANM Annual Congress in Barcelona, and here we are. Six reviews deal with 20 Patient Intervention Comparison Outcome (PICO) questions and treat the clinical and pre-clinical stages of the main dementing neurodegenerative disorders. The seventh review is devoted to the question of automatic quantification assessment (PICO 21), and the eighth one describes the methodology followed by the group of facilitators, assisted by the EAN guideline working group and by the panelists.

To summarize the impression we had while working on the various topics, we were surprised by the relatively few papers supporting the evidence for use of FDG-PET in a clinical scenario. The literature is rich, indeed, but seldom the articles satisfy the requisites to be selected as 'paper giving evidence'. Notwithstanding this heavy limitation, the panelists agreed through a Delphi Consensus procedure (four rounds) that FDGPET is clinically useful by adding information to the clinical 
neuropsychological evaluation in most symptomatic stages of neurodegenerative diseases, not in pre-symptomatic states or in disorders, such as ALS and HD, in which other tools seem more convenient. Also, the panelists agreed on the use of automatic semi-quantitative tools to assist the NMP in reporting scans.

Why so many papers but so few satisfying the rules of the evidence-based medicine? First, spontaneous, monocentric studies using costly diagnostic techniques often fail to achieve adequate sample size and thus to substantially contribute to enrich evidence. Second, there is worldwide difficulty in following patients until death and obtain pathological confirmation. Third, unlike radiopharmaceuticals manufactured by pharma companies (e.g., those for amyloid PET), FDG-PET is in some ways an 'orphan drug' since a nuclear medicine site with a cyclotron can easily produce it, and thus no company has interest in funding formal studies. Fourth, although FDGPET is included in the diagnostic workup of dementing disorders in only some European Countries [7], many groups might not acknowledge the need to achieve more evidence because they already consider FDG-PET an effective and established diagnostic tool in these conditions. Indeed, the exam is already included as a main or supportive feature in the diagnostic criteria of many diseases, including dementia with Lewy bodies [8], behavioral variant frontotemporal dementia [9], primary progressive aphasia [10], and PSP [11] while in Alzheimer's disease (AD) it is a recognized marker of downstream neurodegeneration [12]. Then, people say, we all already fruitfully using it in clinical practice, why should we spend money and time to show what we already know? This reticence is further reinforced by the lack in Europe and elsewhere of a structured framework to validate biomarkers for clinical use, as it has been recently outlined [7].

With this work we hope to help the clinical communities of NMP and clinicians managing dementia in their everyday work by giving them the basis of knowledge available as of the end of 2015 together with expert opinions coming from both the neurology and the nuclear medicine fields. We also hope to have shown the frail points of a knowledge that should be increased by further research, and we guess future research ideas can come from reading this issue of the EJNMMI. Also, we hope that some main messages contained in these eight articles are well received by readers when designing their researches, which allows their conclusions to enrich the available evidence.

Adding to the incomplete availability of evidence, there are some key practical issues still to be solved, and procedures needing harmonization. Just to report some examples, at present, the choice of FDG-PET still greatly depends on the availability of local facilities [13] and, when the differential diagnosis includes $\mathrm{AD}$, it is often an alternative to a cerebrospinal biomarker assay, or, more recently, to amyloid PET. Still to date, one of the main factors driving the choice of FDG-PET is the presence of a nuclear medicine unit with expertise in neurology in general, and in neurodegenerative diseases in particular. It is common experience for clinicians to read reports of non-expert nuclear medicine centers containing mistakes in describing findings and conclusions. There is no homogeneous format to report scans; some NMP describe the findings but do not comment on the most likely diagnostic hypothesis the scan is compatible with. In turn, very often the clinician asking for the examination fails to share the diagnostic hypothesis, thus somehow preventing a meaningful answer. Moreover, there is a number of automatic tools for semi-quantification, giving different results, and no defined rule on whether to use or not to use them, which and how [14]. Some NMP use them to assist in reporting, others strictly repeat in the report the findings shown by these tools without reading images with a critical approach.

In conclusion, we have produced what we could call 'interim' suggestions, waiting to re-consider them as soon as new substantial evidence will be added to the literature. A lot of work remains to be done, and still areas of uncertainty and debate need to be overcome.

Enjoy the reading!

\section{Compliance with ethical standards}

Conflict of interest Flavio Nobili: received personal fees and nonfinancial support from GE Healthcare, non-financial support from EliLilly and grants from Chiesi Farmaceutici.

Reinhold Schmidt: has nothing to declare.

Ignasi Carriò: has nothing to declare.

Giovanni B Frisoni is principal investigator of industry-sponsored trials funded by AbbVie, Acadia, Altoida, Amoneta, Araclon, Biogen, Janssen, Novartis, Piramal; and has received funding for investigatorinitiated trials from GE, Piramal, and Avid-Lilly; and has received speaker fees from a number of pharma and imaging companies.

Ethical approval This article does not contain any new studies with human participants or animals performed by any of the authors. The human studies discussed herein come exclusively from previously published research articles.

Informed consent Not applicable, this is an Editorial.

\section{References}

1. Varrone A, Asenbaum S, Vander Borght T, Booij J, Nobili F, Någren $\mathrm{K}$, et al. European Association of Nuclear Medicine Neuroimaging Committee. EANM procedure guidelines for PET brain imaging using [18F]FDG, version 2. Eur J Nucl Med Mol Imaging. 2009;36:2103-10.

2. Filippi M, Agosta F, Barkhof F, Dubois B, Fox NC, Frisoni GB, et al. European Federation of the Neurologic Societies. EFNS task force: the use of neuroimaging in the diagnosis of dementia. Eur J Neurol. 2012;19:1487-501.

3. Nobili F, Festari C, Altomare D, Boccardi M. Incremental value of automated assessment of FDG-PET compared to visual reading, in the diagnostic work-up of patients with dementing neurodegenerative disorders. Eur J Neurol. 2017;24(Suppl 1):611. 
4. Boccardi M, Festari C, Arbizu J, Orini S, Gandolfo F, Altomare D, et al. Diagnostic utility of FDG-PET in detecting Alzheimer's disease in patients with persistent MCI of uncertain origin. Eur J Neurol. 2017;24(Suppl 1):447.

5. Altomare, Festari, Rivolta, Gandolfo, Orini, Drzezga, et al. Diagnostic utility of FDG-PET in identifying AD dementia with atypical presentation or atypical course. Eur J Neurol. 2017;24(Suppl 1):610.

6. Altomare D, Festari C, Walker Z, Rivolta J, Gandolfo F, Orini S, et al. Diagnostic utility of FDG-PET in differentiating between Alzheimer's and Lewy bodies disease. Eur J Neurol. 2017;24(Suppl 1):612.

7. Frisoni GB, Boccardi M, Barkhof F, Blennow K, Cappa S, Chiotis $\mathrm{K}$, et al. Strategic roadmap for an early diagnosis of Alzheimer's disease based on biomarkers. Lancet Neurol. 2017;16:661-76.

8. McKeith IG, Boeve BF, Dickson DW, Halliday G, Taylor JP, Weintraub D, et al. Diagnosis and management of dementia with Lewy bodies: fourth consensus report of the DLB consortium. Neurology. 2017;89:88-100.

9. Rascovsky K, Hodges JR, Knopman D, Mendez MF, Kramer JH, Neuhaus J, et al. Sensitivity of revised diagnostic criteria for the behavioural variant of frontotemporal dementia. Brain. 2011;134: 2456-77.

10. Gorno-Tempini ML, Hillis AE, Weintraub S, Kertesz A, Mendez M, Cappa SF, et al. Classification of primary progressive aphasia and its variants. Neurology. 2011;76:1006-14.

11. Boxer AL, Yu JT, Golbe LI, Litvan I, Lang AE, Höglinger GU. Advances in progressive supranuclear palsy: new diagnostic criteria, biomarkers, and therapeutic approaches. Lancet Neurol. 2017;16:552-63.

12. Albert MS, DeKosky ST, Dickson D, Dubois B, Feldman HH, Fox $\mathrm{NC}$, et al. The diagnosis of mild cognitive impairment due to Alzheimer's disease: recommendations from the National Institute on Aging-Alzheimer's Association workgroups on diagnostic guidelines for Alzheimer's disease. Alzheimers Dement. 2011;7:270-9.

13. Riello R, Albini C, Galluzzi S, Pasqualetti P, Frisoni GB. Prescription practices of diagnostic imaging in dementia: a survey of 47 Alzheimer's Centres in Northern Italy. Int J Geriatr Psychiatry. 2003;18:577-85.

14. Frisoni GB, Bocchetta M, Chételat G, Rabinovici GD, de Leon MJ, Kaye J, et al. ISTAART's NeuroImaging professional interest area. Imaging markers for Alzheimer disease: which vs how. Neurology. 2013;81:487-500. 